\title{
210 Congenital laryngeal web
}

A Symptoms may be absent.

B Usually involves the posterior one sixth of the vocal cords.

C The voice is of normal quality.

D Excision should be performed as early as possible.

E The atresia may be a cause of still birth.

\section{In closed laryngeal injuries}

A Surgical emphysema can involve the chest and abdomen.

$B$ The cricoid cartilage is most frequently fractured.

C A fixed vocal cord is always due to damage to the recurrent laryngeal nerve.

D Dyspnoea is due to pressure of the cervical vertebrae on the larynx.

E Perichondritis may be prevented by performing urgent tracheostomy. 